\title{
Motion Artefact in Voltage-Sensitive Fluorescent Dye Emission During Repeated Ischemia of Isolated Heart
}

\author{
O. JANOUŠEK ${ }^{1,2}$, J. KOLÁŘOVÁ ${ }^{1,2}$, M. RONZHINA ${ }^{1,2}$, M. NOVÁKOVÁ ${ }^{3}$, \\ I. PROVAZNÍK ${ }^{1,2}$
}

${ }^{1}$ Department of Biomedical Engineering, Faculty of Electrical Engineering and Communication, University of Technology, Brno, Czech Republic, ${ }^{2}$ International Clinical Research Center Center of Biomedical Engineering, St. Anne's University Hospital Brno, Brno, Czech Republic, ${ }^{3}$ Department of Physiology, Faculty of Medicine, Masaryk University, Brno, Czech Republic

Received December 22, 2011

Accepted February 12, 2013

On-line April 16, 2013

\begin{abstract}
Summary
Motion artefact (MA) in voltage-sensitive fluorescent signals causes significant debasement of action potential. During ischemia and reperfusion in isolated rabbit heart, this artefact develops in a manner which may be described by the time of its onset, level, and shape. The MA during ischemia: (a) may become substantial with approximately two minutes delay after establishing global ischemia; (b) may be almost twice as high as the physiological action potential and decreases both with time and repetition of ischemia; (c) the MA shape is unpredictable and depends on individual rabbit.
\end{abstract}

\section{Key words}

Motion artefact • Ischemia • Isolated rabbit heart • Voltagesensitive dye

\section{Corresponding author}

O. Janoušek, Department of Biomedical Engineering Faculty of Electrical Engineering and Communication, Brno University of Technology, Kolejní 4, 61200 Brno, Czech Republic. E-mail: xjanou12@stud.feec.vutbr.cz

\section{Introduction}

The spread of excitation within the cell membranes is a complex biophysical phenomenon with functional consequences in various cells and/or tissues. In excitable cells, the action potential (AP) represents the signaling mechanism for cell-to-cell communication and the trigger for important cellular functions, e.g. muscle contraction (Salama and Choi 2000). Physiological and biochemical processes inside the cells may be assessed by AP's kinetics, shape, magnitude, and duration.

Optical method represents a promising tool for electrophysiology studies. Optical method has become an important way how to study electrical propagation during last few decades (Cohen and Salzberg 1978, Mironov et al. 2006). This approach uses voltage-sensitive dyes (VSD) (Girouard et al. 1996). VSD is a special chemical compound which binds to the cardiac cell membrane and responds to the changes of surrounding electrical field by change of its optical attributes. VSD in the tissue is excited and produces emission light which reflects electrical activity of cell membrane. This light is collected by photo-detector and evaluated in $\mathrm{mV}$ of membrane potential. Thus, these compounds serve as microscopic voltage-optical transducers. VSDs provide a powerful new technique for action potential recording in systems where the use of electrodes is inconvenient or impossible (Nováková et al. 2008). Usage of VSD allows touch-less recording and eliminates restrictions associated with placing of electrodes to the tissue. In cardiac studies, optical method is frequently used for long-time recordings (up to 1.5 hour) from the same place of the heart.

However, APs recorded by the optical method may be significantly disturbed by the motion artifact (MA). Devaluation of AP by MA is currently the most serious disadvantage of optical method (Himel and Kinsley 2006). 
MA is described as any unwanted distortion of the AP shape caused by the heart movement. MA origin hasn't been fully elucidated yet. Numerous factors may contribute to MA formation. Probably the most important one is the increment of the distance between the photodetector and the heart's wall during cardiac contraction. Changed distance results in total fluorescence modulation and consequently inaccurate shape of recorded AP. Its shape is also affected by changes in total number of VSD molecules observed by photo-detector during heart contraction. Besides two abovementioned factors several other phenomena disturb AP, such as inhomogeneous placement of VSD molecules in tissue, and reflectance, dispersion, absorption of the light in tissue and an exhaustive enumeration of all disturbing factors has not been published yet.

Removing, or at least suppression, of motion artefact is currently achieved by several methods (Knisley et al. 2000): use of pharmacological blockers of muscle contraction, mechanical restriction of the heart (Nygren et al. 2002, Li and Nattel 2007), decreasing of extracellular calcium concentration ( $\mathrm{Li}$ and Nattel 2007), and signal processing techniques, especially ratio method (Knisley et al. 2000) and subtraction method (Tai et al. 2004). None of these approaches, except signal processing methods, seems to be ideal because their use may affect physiological behaviour of the tissue or the whole organ. The ratio and subtraction methods overcome this problem. Recently insufficiency of ratio method has been reported in cases where MA was substantial in optically recorded AP (Tai et al. 2004). However, this method is optimal for suppression of small MA. This indicates that knowledge of MA behavior during ischemia repetition may improve noticeably choice of optimal MA suppression method.

It is not known yet whether and how MA develops during long-time recording and whether changes in cardiac electrophysiology or experimental conditions may affect its characteristics. It is supposed that energetic exhaustion of the heart, caused by ischemia, seriously affects motion of the isolated heart, and thus, consequently, MA.

In our laboratory, APs in isolated mammalian hearts are recorded by optical method using VSD di-4-ANEPPS under various physiological and pathophysiological conditions. APs are generally disfigured by MAs which affect the shape of APs and impede their analysis. Therefore, the aim of this study was to describe the MA changes in spontaneously beating isolated rabbit hearts during long-time recording accompanied by repeated episodes of flow ischemia.

\section{Materials and Methods}

\section{Experimental overview}

Twelve New Zealand white rabbits were included in this study. After premedication with benzodiazepines (Apaurin, 2 mg, i.m., Krka, Slovenia), the animals were deeply anaesthetized by mixture of ketamin $(60 \mathrm{mg} / \mathrm{kg}$ of body mass, Narkamon, Spofa, Czech Republic) and xylazin (2 mg/kg of b.m., Rometar, Spofa, Czech Republic), artificially ventilated and the chest was opened. Then the heart was excised with a sufficiently long segment of ascending aorta. The aorta was cannulated, the heart mounted on the modified Langendorff apparatus (Nováková et al. 2000) and placed in thermostat-controlled bath $\left(37^{\circ} \mathrm{C}\right)$ filled with KrebsHenseleit solution of following composition (in $\mathrm{mM}$ ): $\mathrm{NaCl} 118, \mathrm{NaHCO}_{3} 24, \mathrm{KCl} 4.2, \mathrm{KH}_{2} \mathrm{PO}_{4}$ 1.2, $\mathrm{MgCl}_{2}$ 1.2, glucose 5.5, Taurine 10 , and $\mathrm{CaCl}_{2}$ 1.2. The solution was oxygenated with $95 \% \mathrm{O}_{2}$ and $5 \% \mathrm{CO}_{2}$. The hearts were perfused in the mode of constant perfusion pressure ( $85 \mathrm{~mm} \mathrm{Hg}$ ) and stabilized for $30 \mathrm{~min}$. Electrical activity of the heart was recorded simultaneously by electrode system and by optical method.

\section{Orthogonal electrogram system}

The heart was placed into sufficiently large transparent Plexiglas bath filled with Krebs-Henseleit solution. Electrogram signals (EGs) were recorded by six $\mathrm{Ag}-\mathrm{AgCl}$ disc electrodes representing three bipolar leads in orthogonal directions ( $\mathrm{x}, \mathrm{y}$, and $\mathrm{z}$ ). All electrodes were adjustable and passed through Plexiglas wall of the bath, four from the sides, one from the bottom, and one from the top cover of the bath. Each heart included in this study was positioned in the bath in the same way. Isolated heart was beating freely and it was not mechanically restricted.

The EGs were recorded by touch-less method (Nováková et al. 2000) with data acquisition multifunction card PCI-6111E (National Instruments, USA) with sampling frequency $\mathrm{fs}=2000 \mathrm{~Hz}$. EG signals were acquired by designed application in LabView 7.1 software (National Instruments, 2008). Analogue to digital conversion was performed using a 12-bit A/D converter. The digital signal was stored on a hard disk for off-line processing. Three orthogonal EGs signals with duration approximately two hours were recorded. 
Afterwards, 10 minutes-long segments of the signal were processed in Matlab R2006a (MathWorks, 2006).

\section{Optical method for AP recording}

APs were recorded by touch-less optical method with di-4-ANEPPS, most commonly used dye in cardiac studies (Salama 2001). Excitation was produced by halogen light (Volpi AG, Switzerland) with optical filter $(\lambda=506 \pm 30 \mathrm{~nm})$ and transferred to the heart wall by an optical probe (Avantes, Netherlands). The optical probe passed through Plexiglas bath and ended in proximity of the left ventricular wall. Micromanipulator allowed to set-up the probe in a position which was close to the heart wall but did not restrict movement of the beating heart (Provazník et al. 2003). The same optical probe was used for transfer of emitted light to the photo detector (6 of 7 optical fibres in this multi-fibre probe were used for excitation light, 1 was used for emission light). A photodiode (Tesla, Czech Republic) was used as a photo detector.

\section{Experimental protocol}

Experiment, designed for studies of isolated heart preconditioning, consisted of ten phases. In the first phase, each heart was placed into the bath immediately after preparation and underwent stabilization period; its rhythm was stabilized and eventually the heart exhibiting any arrhythmias was excluded. In the second phase, the heart was loaded with VSD for $15 \mathrm{~min}$. VSD was diluted in $\mathrm{K}-\mathrm{H}$ solution and applied to coronary arteries. During the third phase - 15 min washing - redundant noncoupled VSD molecules were removed. The fourth phase was 30 min long control period during which control APs were recorded. Next six phases were represented by 10 min periods of flow (global) ischemia and reperfusion interchanging. During control and VSD loading phase of experiment only EGs were recorded, whereas EGs with APs were recorded during the last six phases of experiment. Recorded APs were substantially disturbed by MA, however.

\section{AP model and its fitting}

For the purpose of MA analysis, the AP was subtracted from recorded optical signal, so that only MA remained for detailed analysis. The model of single AP (mAP) was created and used for subtraction. Model of single AP was created as an average optical signal from the control period where MA was negligible and AP was stable and without variation. It was created for each individual animal included in this study because APs of individual subjects had slightly different heights of upstroke and durations of AP due to variability caused by placement of optical probe to the tissue and interindividual differences in heart structure.

Subtraction required fitting of mAP curve to real signal as pre-processing step. Three important markers in mAP signals were utilized for the fitting mAP to real signals:

1. AP upstroke (marked by number 1 in Fig. 1A). The AP upstroke was defined as position of the maximum of the first derivative of the AP. This parameter had never been affected by MA. Placement of the first marker works well for real optical signals even in the presence of noise (see Fig. 1B).

2. Height of AP (marked by number 2 in Fig. 1A). The height of AP was defined as a height of AP plateau in point $20 \mathrm{~ms}$ after AP upstroke occurrence. Height of AP was never affected by MA.

3. AP downstroke (marked by number 3 in Fig. 1A). The AP downstroke was defined as a time at which AP reached basic isoline. This time was defined as position of maximum of $\mathrm{T}$-wave in simultaneously recorded EG. MA influenced downstrokes of recorded APs significantly which impede determination of AP downstroke placement directly from AP. T-wave maximum and AP downstroke occurs at the same time, however. It holds even in presence of ischemia, as may be seen in Figure 2. This fact has been recently published in Kolářová et al. (2010).

Height and duration of mAP has been adjusted to each repetition of recorded AP before mAP subtraction.

\section{Results}

Optical signals and orthogonal EG signals were recorded from surface of 12 isolated rabbit hearts during control, ischemia and reperfusion periods. Optical signals consist of series of repetition of AP. AP repetitions were averaged before subsequent analysis for signal-to-noise ratio (SNR) improvement. Total number of 41 AP repetitions gave the highest SNR of averaged AP. SNR of single AP was $6.83 \mathrm{~dB}$ and $\mathrm{SNR}$ of averaged AP was $44.55 \mathrm{~dB}$, respectively. Averaged AP from control period has 6.5 times higher SNR than single AP, whereas kinetics and shape of AP remain unaltered.

The model AP was subtracted from each averaged AP subsequently and respective MA remained to detailed analysis. 

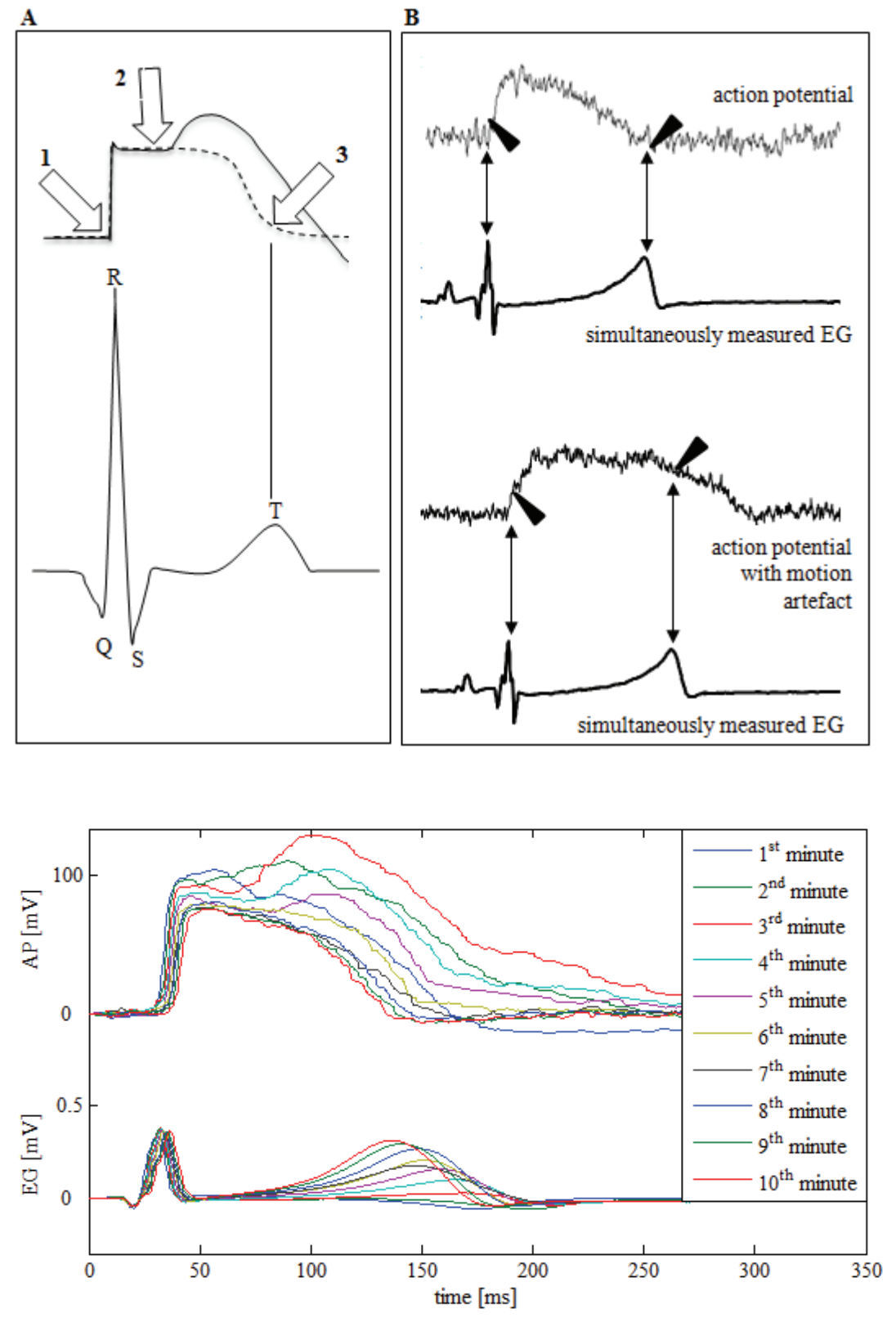

Fig. 1. (A) AP upstroke (arrow 1) detection was based on first derivative of AP curve. Detection of AP downstroke was based on EG curve analysis with T-wave maximum assessment (arrow 3). (B) Detection of upstroke and downstroke of AP works both for signal without motion artefact and signal with motion artefact in real data. Black wedges represent detection of AP upstroke and AP end.

Fig. 2. The simultaneous recording of EGs and APns during ten minutes of ischemia period, reprinted with permission from Kolářová et al. (2010).
It has been found that the MA shape in individual AP records differs. Due to enormous number of analysed MA (over 5000) and because of high interindividual MA shape variability it is hardly possible to present each shape of MA devaluation. Therefore, two parameters - mass of MA and maximum of MA - were computed for each single MA and their evolution during repeated ischemia and reperfusion periods was analysed. Mass of MA was calculated as integral of MA in the range determined by AP duration. For the purpose of MA mass comparison in multiple records each MA mass was expressed as percentual change against MA mass in control period:

$$
M A_{m}=\frac{O P T_{m}-m A P(0)}{m A P(0)} \cdot 100[\%]
$$

where $\mathrm{MA}_{\mathrm{m}}$ is $\mathrm{MA}$ in the $m$-th minute of experiment phase, $\mathrm{OPT}_{\mathrm{m}}$ is optically recorded signal composed from MA and AP in the $m$-th minute of experiment phase, and $\operatorname{mAP}(0)$ is model of AP created from AP records in the control phase of particular experiment.

Evolution of mass is presented in form of its mean and variance in Figure 3. Thick line shows mean of MA mass during six phases of experiment. Evolution of MA during ischemia has specific shape, observable in all three ischemic phases. Comparison between ischemic phases revealed that repetition of global ischemia decreases MA level, nevertheless specific shape of MA evolution remains uniform. MA is substantial between the second and the third minute of each ischemic phase. MA remains non-substantial and small during reperfusion 

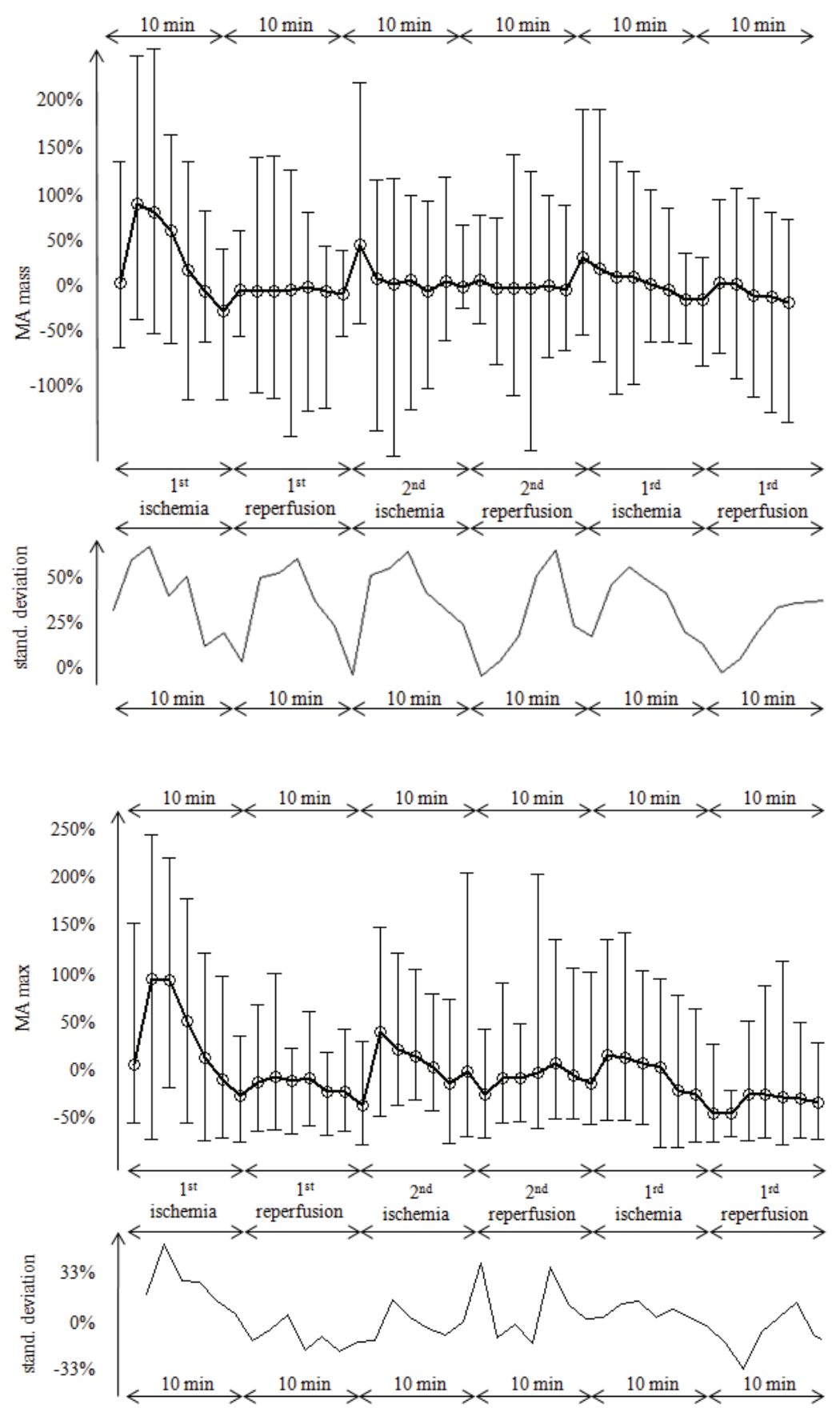

Fig. 3. MA mass in ischemic and reperfusion periods. Mean value of MA mass (thick line, upper part of figure) shows that MA mass is substantial in initial phase of ischemia, especially between the second and the third minute of ischemia duration. Mean value of MA mass has specific evolution, conspicuous in each ischemic period. Standard deviation of MA mass (bottom part of figure) shows that the lowest MA mass variance is located at the end of ischemic phases.
Fig. 4. Maximum of motion artifact in ischemic and reperfusion periods. Mean value of MA maximum (thick line) shows that MA is substantial in initial phase of ischemia, especially between the second and the third minute of ischemia duration. Mean value of MA maximum has specific evolution, conspicuous in each ischemic period; however, its shape is not so smooth as for mass of MA. As well as in MA mass, individual behaviour of motion artefact in all twelve measured signals has enormous variance which impede to usage of mean value for automatic classification purposes. Standard deviation of MA maximum is shown in bottom part of picture. periods. On the other side, not only mean value describes adequately behaviour of MA during ischemia. Variance of MA mass in individual recordings has to be taken into account too. Variance of MA mass in analysed signals significantly changes during the experiment. Standard deviation of MA mass is shown in bottom part of Figure 3. The shape of MA mass variance is not constant; the lowest values are observable at the end of ischemic phases and at beginning of reperfusion phases.

Maximum of MA was calculated as maximum value of MA in the range determined by AP duration. Maximum of MA was recalculated to percentual change against control period - the same as for mass of MA - and presented in the form of mean and variance in Figure 4. Thick line shows mean of MA maximum. Maximum of MA has similar shape as mass of MA during ischemic periods; however, the curve is less smooth than curve of MA mass in reperfusion periods. Comparison between ischemic phases shows that the second repetition of global ischemia decreases the MA level significantly, whereas there is almost none difference between the second and the third ischemic period. The difference between ischemia and following reperfusion is striking only in case of the first ischemia and reperfusion, whereas there is only a slight difference between the second ischemia and its reperfusion, as well 


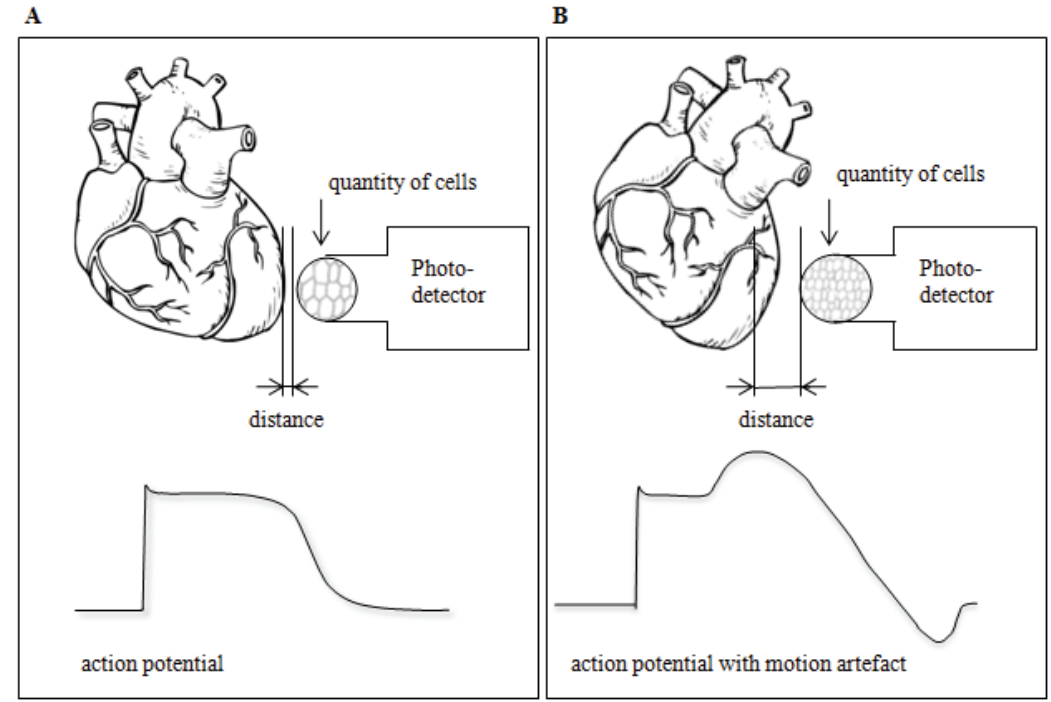

Fig. 5. Action potential without motion artefact (A) and with motion artefact (B). Shape of action potential is influenced by changes in distance of the heart wall from photo-detector. Action potential shape is also altered by changes in number of cells in photo-detector's field-of-view, caused by contraction of heart.

as between the third ischemia and its reperfusion.

Variance of MA max in individual signals is large. Standard deviation of MA maximum is shown at bottom part of Figure 4. The shape of standard deviation is not constant, similarly as in case of MA mass; however, there is no visible pattern common for all ischemic phases.

\section{Discussion}

In our experiments on isolated rabbit hearts, motion artefact evolution was observed in optically recorded action potentials during repeated ischemia. Influence of time and global ischemia repetition on motion artefact was studied.

Results of this study show that MA shape in individual optical signals is unpredictable and depends on each particular heart. Various shapes of MA in individual subjects obstruct automatic classification of MA level. Based on our study, we conclude that two parameters mass of MA and maximum of MA - represent better parameters for MA level description than MA shape. These parameters might be used for classification of MA level and may help with the right choice of MA suppression method.

In this study, it was observed that evolution of both parameters is specific during ischemia. Shape of MA evolution curve is preserved in each ischemic phase, although its amplitude decreases with ischemia repetitions.

Besides decrement of MA level caused by ischemia repetition, also MA level changed with time. MA significantly varied during time: it became substantial not immediately after introducing global ischemia, but approximately after two minutes of its onset. MA shape in individual records remained unpredictable. Some recorded MAs were almost twice higher than respective APs, whereas others were negligible. Generally it can be concluded, that MA decreased with time. This fact is obvious from evolution curve of MA mass and MA max parameters.

The decrement of MA level during time and during repeated ischemia periods can be attributed to energetic depletion of ischemic tissue. The MA in our study was recorded with use of voltage-sensitive dye (VSD). Contraction of heart causes prolongation of the distance between the heart wall, dyed with VSD, and photo detector. Prolonged distance decreases a total light recorded by photo-detector, which leads to MA. This phenomenon is illustrated in Figures 5A and 5B. Degree of contraction becomes reduced at the end of each ischemic phase due to insufficient amount of nutrition and oxygen.

Moreover, any contraction of myocardium causes reduction of heart volume. Contraction of cardiomyocytes increases their number in the field of photo-detector view. As a consequence, more molecules of VSD produce more light emission which leads to nonlinear modulation of measured signal. Comparison between contracted and non-contracted heart is presented in Figures $5 \mathrm{~A}$ and $5 \mathrm{~B}$. The energetic depletion of the isolated heart causes lowering of the contraction level, which leads to smaller changes in MA. As a result, the MA level decreases during the time. Both abovementioned phenomena form shape of MA, as can be seen in the bottom part of Figures $5 \mathrm{~A}$ and $5 \mathrm{~B}$.

Moreover, other factors affect the shape of motion artefact, such as altered optical path caused by 
changes in reflectance, scattering, and absorbance (Knisley et al. 2000) caused by changes in geometry of measured tissue. Altered spatial geometry of VSD may also contribute to MA formation (Rosenbaum and Jalife 2001) in some cases, but this phenomenon did not influenced MAs in this study. In our experiments, di-4-ANEPPS was used. This VSD belongs to electrochromism group of VSD where spatial geometry of VSD is insignificant for MA origin.

Based on our results it can be concluded that MA shape cannot be generally described due to enormous inter-individual variation of MA in studied signals. Furthermore, it can be concluded that MA level is diminished after each global ischemia intervention, and evolution of MA during ischemic phases has a specific shape with maximum of MA after two minutes of ischemic onset.

The limitation of this study may be seen in AP fitting procedure. It should be noted that fitting procedure is based on assumption that ischemia affects AP upstroke and AP duration only and AP shape remains unaltered. Persistency of the AP shape in ischemic conditions has been observed previously (Shaw and Rudy 1997). On the other hand, some accidental changes of AP, such as triangulation of AP or early afterdepolarization, might degrade presented results.

\section{Conflict of Interest}

There is no conflict of interest.

\section{Acknowledgements}

All experiments followed the guidelines for animal treatment approved by local authorities and conformed to the EU law. This work was supported by the grant projects of the Grant Agency GACR 102/12/2034, GACR 102/09/H083, and MUNI/A/0951/2012. Supported by European Regional Development Fund - Project FNUSA - ICRC (No. CZ.1.05/1.1.00/02.0123).

\section{References}

COHEN LB, SALZBERG BM: Optical measurement of membrane potential. Rev Physiol Biochem Pharmacol 83: $35-$ $88,1978$.

GIROUARD SD, LAURITA KR, ROSENBAUM DS: Unique properties of cardiac action potentials recorded with voltage-sensitive dyes. J Cardiovasc Electrophysiol 7: 1024-1038, 1996.

HIMEL HD, KNISLEY SB: Imaging of cardiac movement using ratiometric and nonratiometric optical mapping: effects of ischemia and 2, 3-butaneodione monoxime. IEEE Trans Med Imaging 25: 122-127, 2006.

KNISLEY SB, JUSTICE KR, KONG W, JOHNSON PL: Ratiometry of transmembrane voltage-sensitive fluorescent dye emission in hearts. Am J Physiol Heart Circ Physiol 279: 1421-1433, 2000.

KOLÁŘOVÁ J, FIALOVÁ K, JANOUŠEK O, NOVÁKOVÁ M, PROVAZNÍK I: Experimental methods for simultaneous measurement of action potentials and electrograms in isolated heart. Physiol Res 59 (Suppl 1): 71-80, 2010.

LI D, NATTEL S: Pharmacological elimination of motion artifacts during optical imaging of cardiac tissues: Is blebbistatin the answer? Heart Rhythm 4: 627-628, 2007.

MIRONOV S, VETTER FJ, PETRSOV AM: Fluorescence imaging of cardiac propagation: spectral properties and filtering of optical action potentials. Am J Physiol Heart Circ Physiol 291: H327- H335, 2006.

NOVÁKOVÁ M, MOUDR J, BRAVENÝ P: A modified perfusion system for pharmacological studies in isolated hearts. In: Analysis of Biomedical Signals and Images. 15th Biennial International Eurasip Conference Biosignal 2000. Vyd. 1. Vutium Press, Brno, 2000, pp 162-164.

NYGREN A, KONDO C, CLARK RB, GILES WR: Voltage-sensitive dye mapping in Langendorff-perfused rat hearts. Am J Physiol Heart Circ Physiol 284: 892-902, 2003.

PROVAZNÍK I, NOVÁKOVÁ M, VESELÝ Z, BLAHA M, CHMELǍ̌ M: Electro-optical recording system for myocardial ischemia studies in animal experiments. In: Computer in Cardiology. Danvers, USA, 2003, pp 573537.

ROSENBAUM DS, JALIFE J: Response of cardiac myocytes to electrical fields. In: Optical Mapping of Cardiac Excitation and Arrhythmias. Futura Publishing Company, Inc., New York, 2001, pp 313-333. 
SALAMA G: Optical mapping: background and historical perspective. In: Optical Mapping of Cardiac Excitation and Arrhythmias. DS ROSENBAUM, J JALIFE (eds), Futura Publishing Company, Inc., New York, USA, 2001, pp 9-31.

SALAMA G, CHOI BR: Images of action potential propagation in heart. News Physiol Sci 15: 33-41, 2000.

SHAW RM, RUDY Y: Electrophysiologic effects of acute myocardial ischemia: a theoretical study of altered cell excitability and action potential duration. Cardiovasc Res 32: 256-272, 1997.

TAI DC, CALDWELL BJ, LEGRICE IJ, HOOKS DA, PULLAN AJ, SMAILL BH: Correction of motion artefact in transmembrane voltage-sensitive fluorescent dye emission in hearts. Am J Physiol Heart Circ Physiol 287: H985-H993, 2004. 